\title{
Exploring Stroke Outcomes Following a Door-to-Needle Quality Improvement Project
}

\author{
Susan Alcock, Jo-Ann V. Sawatzky, Trevor Strome, Kathy Doerksen
}

\begin{abstract}
Background: Although stroke rates in Canada are expected to increase dramatically over the next decade, time-driven hyperacute stroke care with thrombolysis increases the likelihood of a good clinical outcome. Following a period of suboptimal performance results for stroke care, our tertiary care center undertook a door-to-needle (DTN) quality improvement initiative. The purpose of our study was to determine if the resulting improved median DTN times and greater proportion of patients treated within 60 minutes of arrival at our emergency department were associated with improved clinical outcomes. Methods: Guided by the Donabedian quality framework, we retrospectively reviewed charts of consecutive patients $(n=324)$ who received thrombolysis preand post-quality improvement initiative. Data on patient characteristics, and process and outcome measures were collected. Primary study outcomes included mortality, adverse events, discharge location, and independence at discharge. Data analysis compared proportions with Chi Square and means using the two-tailed $t$-test and a 0.05 level of significance. Results: Median DTN times and the percentage of cases with a DTN $\leq 60$ minutes improved significantly post-intervention $(p<0.001)$. In-hospital mortality decreased $(p=0.013)$, and the proportion of favorable versus unfavorable discharge locations improved $(p=0.005)$. Mortality rates for all study patients with DTN $\leq 60$ versus $>60$ minutes were also significantly lower $(p=0.044)$ post-intervention. Conclusions: Our quality improvement initiative resulted in timelier care and positively influenced clinical outcomes. This study highlights the need for ongoing, innovative investment strategies to ensure timely hyperacute stroke care and optimal patient outcomes.
\end{abstract}

RÉSUMÉ : Évolution de l'état de santé de patients victimes d'un AVC dans le cadre d'un projet d'amélioration de la qualité des soins Contexte : Bien que l'on s'attende à ce que les taux d'AVC au Canada augmentent de manière spectaculaire au cours de la prochaine décennie, le fait de prodiguer en temps opportun un traitement thrombolytique dans des cas d'AVC hyper-aigus augmente la probabilité que l'évolution du patient soit favorable sur le plan clinique. À la suite d'une période en vertu de laquelle notre performance en matière de soins destinés à des victimes d'un AVC était sous-optimale, notre centre de soins tertiaires a décidé de mettre sur pied un projet d'amélioration de la qualité de ces soins en fonction de la variable « arrivée-injection » (door-to-needle). L'objectif de cette étude a donc été de déterminer dans quelle mesure l'amélioration de cette variable et une plus grande proportion de patients traités dans un délai de 60 minutes étaient associées à une amélioration de l'évolution de leur état de santé. Méthodes : Au moyen du modèle de Donabedian, nous avons passé en revue de manière rétrospective les dossiers de patients vus consécutivement $(\mathrm{n}=324)$ ayant bénéficié d'un traitement thrombolytique avant et après la mise sur pied de notre projet. Nous avons ainsi collecté des données portant sur les caractéristiques de ces patients, sur les processus entre leur arrivée et leur prise en charge mais aussi sur l'évolution de leur état de santé. Les principaux résultats de cette étude ont inclus leurs taux de mortalité, la présence d'événements indésirables, leurs destinations à la suite d'un congé et leurs niveaux d'autonomie une fois ce congé obtenu. Notre analyse de données a comparé des proportions au moyen du test du $\chi^{2}$. Elle a aussi comparé des moyennes en utilisant le test de Student bilatéral et un degré de signification de 0,05. Résultats : Les délais médians entre l'arrivée des patients et un traitement thrombolytique, de même que le pourcentage de cas dont ces délais étaient inférieurs à 60 minutes, se sont améliorés de manière notable à la suite de notre projet $(p<0,001)$. Ajoutons aussi que le taux de mortalité hospitalière a diminué $(p=0,013)$ et que la proportion des destinations dites favorables a augmenté $(p=0,005)$. Enfin, les taux de mortalité de tous les patients dont les délais pour une première injection étaient inférieurs à 60 minutes se sont révélés notablement plus bas que ceux des patients dont les délais dépassaient les 60 minutes $(p=0,044)$. Conclusion : Il s'avère que notre projet s'est traduit par un accès plus rapide à des soins, ce qui a eu un impact positif sur l'évolution de l'état de santé des patients. Cette étude met donc en perspective le besoin de stratégies d'investissement permanentes et innovantes afin d'assurer une prise en charge en temps opportun des patients victimes d'un AVC et une évolution optimale de leur état de santé.

Keywords: Door-to-needle, Quality improvement, Clinical outcomes, Stroke

doi: $10.1017 / \operatorname{cjn} .2020 .7$

Can J Neurol Sci. 2020; 47: 167-175

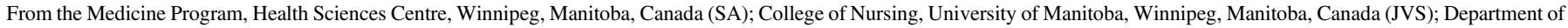

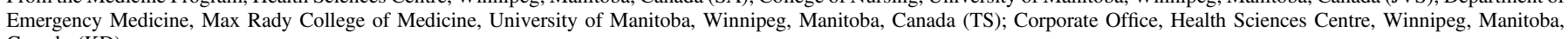
Canada (KD)

Received December 5, 2018. Final Revisions Submitted December 28, 2019. Date of Acceptance January 1, 2020.

Correspondence to: Susan Alcock, Health Sciences Centre, 820 Sherbrook St., Winnipeg, Manitoba, R3A 1R9, Canada. Email: salcock@exchange.hsc.mb.ca 


\section{INTRODUCTION}

Approximately, 62,000 Canadians are diagnosed with stroke each year. ${ }^{1}$ Moreover, the impact of stroke in Canada is devastating. Despite treatment advancements, acute stroke remains the third leading cause of death and the leading cause of severe disability in Canada. ${ }^{1}$ Based on the current trends and projections for an increasingly aging Canadian population, ${ }^{1,2}$ stroke rates are expected to double in the next 20 years. ${ }^{1}$

Today, major stroke is very treatable if the victim arrives at a hospital that offers hyperacute stroke care within the recommended treatment windows. Current reperfusion therapies for acute ischemic stroke, including thrombolytic therapy and mechanical clot retrieval, are time-sensitive; hence, the earlier the treatment is established, the greater the benefit. With every minute that passes, the average acute stroke patient loses 1.9 million neurons, 13.8 billion synapses, and $12 \mathrm{~km}$ of axonal fibers. ${ }^{3}$ Unfortunately, hospital arrival time for more than half of Canadian stroke victims is over 6 hours, which is well beyond the 4.5-hour window of opportunity in which to deliver thrombolytic therapy. Moreover, median door-to-needle (DTN) times of 90 minutes ${ }^{4}$ suggest that treatment on arrival at the hospital is not quick enough. ${ }^{5}$ Hence, barriers to optimal stroke care continue to be pervasive within the prehospital and emergency department (ED) settings.

Evidence-based hyperacute guidelines, for both pre-hospital and ED care, have been developed to minimize barriers to care. .,6 $^{1,6}$ These guidelines focus on rapid assessment, diagnostics, and treatment initiation. ${ }^{1,6}$ Measuring hyperacute process indicators can identify the sources of delays. Importantly, there is now convincing evidence that shorter time intervals from symptom onset to treatment and from ED arrival to treatment result in improved clinical outcomes. ${ }^{1,6-9}$ While several centers have improved their quality stroke care indicators by focusing on improving processes of care, ${ }^{4}$ translating process improvements into patient outcomes remains challenging.

Therefore, using the Donabedian quality framework, ${ }^{10}$ the aim of our study was to explore how the structural changes (i.e., our quality improvement project), which resulted in dramatic process improvements (i.e., reduction in median DTN and increased proportion of patients treated within 60 minutes), impacted patient outcomes (i.e., in-hospital mortality, adverse events, discharge location, and independence at discharge from our hospital). We hypothesized that the significant reduction in median DTN and the increased proportion of patients that received recombinant tissue plasminogen activator (rt-PA) within 60 minutes resulted in improved clinical outcomes.

\section{Methods}

\section{Study Design and Framework}

Our retrospective chart review study was operationalized using the Donabedian framework. ${ }^{10}$ This dominant paradigm in quality of care provides a systematic approach for program evaluation and research. The Donabedian framework focuses on three domains: structure, process, and outcomes, and examines the linkage between the domains. According to this framework, implementing evidence-based structural changes, which result in efficient processes of care, should lead to improved clinical outcomes. In our study, structure represented the setting, or physical environment in which the care took place; operational elements, such as the order sets; provider factors, such as knowledge about hyperacute stroke care; and patient factors, such as stroke risk factors. Process denoted the care provided during the stroke protocol and was measured by well-recognized key stroke quality metrics, such as median DTN times. Outcomes represented patient clinical outcomes, such as mortality and functional outcomes.

\section{Setting and Sample}

The setting for this study was the Health Sciences Centre (HSC), a large tertiary teaching hospital located in Winnipeg, Canada. HSC is the provincial endovascular center for stroke and one of two designated hyperacute stroke centers in Winnipeg that offer rt-PA. During the time of the study, HSC Adult ED treated approximately 250-300 stroke protocols per year. The study population included all consecutive acute ischemic stroke patients who received intravenous rt-PA within the thrombolytic treatment window at the HSC Adult ED between January 1, 2008 and December 31, 2014. Since the intervention was implemented in early 2011, this was considered the washout year and is therefore not included in the analysis.

\section{Quality Improvement Initiative}

Our quality improvement initiative was implemented over a 5-month period in 2011. The initiative incorporated three distinct elements: education, performance feedback, and workflow. Details of the initial education and performance feedback phase of the initiative and the resulting efficiency in the processes of care are described elsewhere. ${ }^{11}$ Education and performance feedback were provided to the neurologists, ED physicians, nurses, unit clerks, and unit assistants, computed tomography (CT) technologists, and laboratory technologists. Our multidisciplinary stroke team received stroke education that included information about the physiological changes that occur in the initial hours following stroke symptom onset; physiological monitoring, in particular the importance of adhering to blood pressure (BP) parameters; core concepts of hyperacute stroke care; the role of each stroke team member during a stroke protocol, and most importantly, why stroke is a time-driven emergency. Performance feedback included process metric results (e.g., median DTN time, median door-to-CT (DTCT), and percentage of patients receiving rt-PA within 60 minutes of arrival).

An interdisciplinary stroke committee was established to address the workflow element of the quality improvement project. A Lean ${ }^{12}$ practitioner played a central role in guiding the team to develop a value stream map (VSM) of an acute stroke patient's pathway through the ED during a stroke protocol. This multidisciplinary team included representatives from the following areas: Winnipeg Fire Paramedic Service, Adult ED (nurses and physicians), Neurology Program, and CT, laboratory, and admitting departments. Based on identified inefficiencies, several workflow processes were adopted/refined. (1) A pre-notification process was already in effect, with Emergency Medical Services (EMS) notifying the ED of an incoming stroke patient; however, the estimated time of patient arrival (ETA) was changed from ETA in number of minutes to specific time of ETA. (2) Code stroke activation processes were refined to ensure that all members of the stroke team received timely notification of the incoming stroke 
protocol. (3) ED patient arrival efficiency was refined so that instead of stopping at triage for registration, all stroke protocol patients are sent directly to a dedicated stroke bed in the resuscitation room, which is adjacent to the CT scanner, thus reducing the transport distance. (4) To improve resuscitation room communication and efficiency, paramedics now give a report to the entire stroke team; the admitting clerk registers the patient; the ED physician completes a primary survey to ensure that the patient's overall status is not compromised; the neurologist does a quick stroke assessment; and one ED nurse establishes intravenous access, while the second nurse documents the patient history. (5) Improved efficiency in diagnostic processes included the CT technologist ensuring that the scanner is ready and notifying the radiologist. Laboratory staff also improved efficiency by preparing for the arrival of the lab specimens. A nurse and the neurologist then escort the patient to the nearby CT scanner. Hence, an efficient, well-orchestrated and coordinated process, with a collaborative team was established.

\section{Data Collection}

Following University of Manitoba ethical review board and institutional access approval, we reviewed the charts of all consecutive patients $(n=324)$ who received thrombolysis pre-quality improvement initiative (January 1, 2008-December 31,$2010 ; n=102$ ) and post-quality improvement initiative (January 1, 2012-December 31, 2014; $n=224$ ). The principal investigator completed all chart reviews. Data collection included demographics, stroke risk factors, presenting symptoms, and baseline clinical characteristics of all participants. Stroke onset time, ED arrival time as documented on the ED record, CT time as noted in nursing record, and time of rt-PA bolus administration or needle time as noted in nursing record were obtained from the patients' charts. Based on these documented times, commonly measured stroke process indicators were calculated: DTN, DTCT, CT-to-needle (CTTN), onset-to-door (OTD), and onset-to-needle (OTN). Outcome data included in-hospital mortality, adverse events (i.e., symptomatic intracranial hemorrhage (SICH) within 36 hours of arrival and life-threatening bleed), discharge location (i.e., home, acute rehabilitation, community hospital, long-term care (LTC) facility, and death), and level of independence at discharge (i.e., independent, ambulatory with assistance, nonambulatory, and death).

\section{Statistical Analysis}

Data were analyzed with IBM ${ }^{\circledR}$ SPSS $^{\circledR}$ Statistics Version 24 (IBM, Armonk, NY, USA). We reported descriptive statistics and explored between-group differences for the pre- and post-quality improvement project phases of this investigation. Discrete variables were analyzed with frequency distributions and percentages. Continuous data were reported with means, medians, standard deviations, and interquartile ranges. Histograms were used to visualize the unique distributions of these data sets. Since data were normally distributed, the $t$-test was used to compare pre- and post-intervention performance for continuous variables. DTN performance data were plotted on run charts to visualize and examine any trends and shifts in performance that occurred during the study period. The Chi-Square test was used to analyze nominal and ordinal scale variables. For variables with small sample sizes (i.e., <5), the Fisher's exact test was used. We also compared clinical outcomes for (i.e., DTN $\leq 60$ vs. DTN $>60$ ) all study patients. The level of statistical significance for all analyses was set at alpha 0.05 .

\section{RESUlts}

\section{Patient Characteristics}

Following data screening, 324 participants were included in the study, with 102 in the pre-intervention group and 222 in the post-intervention group. A comparison of the pre- and post-intervention patient demographics and clinical characteristics are presented in Table 1. Patients' median ages (i.e., 76 and 73 years, respectively) and the proportion of males (i.e., 50\%) were similar in the pre- and post-intervention groups. Stroke risk factors were also similar between the two groups. However, significantly more of the pre-intervention group compared to the post-intervention group were reportedly urban dwellers (88.2\% vs. $64.4 \%$, respectively). As well, patients in the post-intervention group were significantly more likely to arrive by EMS.

Although not statistically significant, slightly more stroke patients arrived during regular daytime hours (i.e., 07:30-17:00) than off hours (57.8\% vs. $54.5 \%)$. Presenting stroke symptoms on ED arrival were also compared, with most patients in both groups presenting with weakness or speech-related symptoms. Patient evaluation factors, including Glasgow Coma Scale scores, baseline systolic and diastolic BP, and blood glucose were similar between groups. Unfortunately, the National Institute of Health Stroke Score (NIHSS) scores were documented in only $2 \%$ of cases in the pre-intervention group and in $28 \%$ of the post-intervention group. Therefore, group comparisons on this variable were not feasible. The proportion of patients in each group with systolic and diastolic BPs above best practice guideline parameters (i.e., a systolic value above 180 or a diastolic value above 105) was also nonsignificant. Notably, the median OTD increased significantly between the preintervention and the post-intervention period (64 vs. 84 minutes; $p<0.001)$.

\section{Process}

The median DTN decreased significantly between the preintervention and the post-intervention period (70.5 vs. 49 minutes; $p<0.001$ ). A run chart (see Figure 1) illustrates DTN time trends and clearly highlights the significant improvement of DTN times following the quality improvement initiative. As expected, a higher number of patients had a median DTN below 60 minutes post-intervention. Median DTCT also exhibited a significant improvement, from 23 minutes in the pre-intervention period to 16 minutes post-intervention $(p<0.001)$. Lastly, the median CTTN for the pre-intervention period was significantly longer compared to the post-intervention period (46.5 vs. 32 minutes; $p<0.001)$.

To determine if our clinical practice reflected best practice, we collected data related to recommendations of the national stroke guidelines $^{1,6}$ that eligible acute stroke patients should receive rt-PA within 30 and 60 minutes of arrival in the ED. A significantly higher percentage of patients in the post-intervention group had a DTN time $\leq 60(p<0.001)$. Based on a Chi-Square analysis, the proportion of stroke patients receiving rt-PA within 


\section{Table 1: Demographics and clinical characteristics of patients with acute ischemic stroke who received rt-PA}

\begin{tabular}{|c|c|c|c|}
\hline Demographic and clinical variables & Pre-intervention $(n=102)$ & Post-intervention $(n=222)$ & $p$-values* \\
\hline $\mathrm{Age}^{\mathrm{a}}$, years & $76(63-84)$ & $73(64-83)$ & 0.786 \\
\hline Male $n(\%)$ & $55(53.9)$ & $111(50)$ & 0.512 \\
\hline Urban $n(\%)$ & $90(88.2)$ & $142(64)$ & $<0.001$ \\
\hline Arrival by EMS $n(\%)$ & $88(86.3)$ & $215(96.8)$ & $<0.001$ \\
\hline Day shift arrival ${ }^{\mathrm{b}}$ & $59(57.8)$ & $121(54.5)$ & 0.574 \\
\hline Onset-to-door time ${ }^{c}$ & $64(44.5-101.5)$ & $84(54.75-137)$ & $<0.001$ \\
\hline \multicolumn{4}{|l|}{ Risk factors } \\
\hline Hypertension $n(\%)$ & $64(62.7)$ & $148(66.7)$ & 0.491 \\
\hline Diabetes $n(\%)$ & $22(21.6)$ & $67(30.3)$ & 0.102 \\
\hline Atrial fibrillation $n(\%)$ & $37(36.3)$ & $67(30.2)$ & 0.275 \\
\hline Current smoker $n(\%)$ & $15(14.7)$ & $31(14)$ & 0.859 \\
\hline Dyslipidemia $n(\%)$ & $25(24.5)$ & $80(36)$ & 0.04 \\
\hline $\mathrm{CAD} / \mathrm{MI} n(\%)$ & $32(31.4)$ & 59 (26.6) & 0.372 \\
\hline Prior stroke or TIA $n(\%)$ & $33(32.4)$ & $68(30.6)$ & 0.756 \\
\hline $\operatorname{PVD} n(\%)$ & $1(1)$ & $5(2.3)$ & $0.669 €$ \\
\hline Carotid stenosis $n(\%)$ & $7(6.9)$ & $8(3.6)$ & 0.195 \\
\hline \multicolumn{4}{|l|}{ Presenting symptoms } \\
\hline Weakness $n(\%)$ & $100(98)$ & $207(93.2)$ & 0.072 \\
\hline Sensory $n(\%)$ & $15(14.7)$ & $23(10.4)$ & 0.259 \\
\hline Balance $n(\%)$ & $9(8.8)$ & $13(5.9)$ & 0.324 \\
\hline Visual $n(\%)$ & $8(7.8)$ & $8(3.6)$ & 0.102 \\
\hline Speech $n(\%)$ & $78(76.5)$ & $170(76.6)$ & 0.983 \\
\hline \multicolumn{4}{|l|}{ Patient evaluation } \\
\hline Admission NIHSS score ${ }^{a}$ & & $14(8-20)$ & \\
\hline Admission GCS ${ }^{\mathrm{a}}$ & $15(12-15)$ & $14(11-15)$ & 0.555 \\
\hline Baseline SBP $^{a}$ & $152(135-170)$ & $153(136-172)$ & 0.684 \\
\hline $\mathrm{SBP}>180 n(\%)$ & $11(10.8)$ & $26(11.8)$ & 0.797 \\
\hline Baseline DBP $^{\mathrm{a}}$ & $82(76-93)$ & $84(7297)$ & 0.587 \\
\hline $\mathrm{DBP}>105 n(\%)$ & $7(6.9)$ & $24(10.9)$ & 0.257 \\
\hline Baseline glucose $\mathrm{a}^{\mathrm{a}}$ & $7.5(2.6)$ & $7.8(2.7)$ & 0.218 \\
\hline
\end{tabular}

EMS $=$ Emergency $\quad$ Medical Services $\quad \mathrm{CAD}=$ coronary artery disease $; \quad \mathrm{MI}=$ myocardial infarction; $\quad \mathrm{TIA}=$ transient ischemic attack; PVD = peripheral vascular disease; NIHSS = National Institute of Health Stroke Score; GCS = Glasgow Coma Scale; $\mathrm{SBP}=$ systolic blood pressure; $\mathrm{DBP}=$ diastolic blood pressure.

Groups compared by $\chi^{2}$ test for categorical variables and $t$-test for continuous variables.

Bold figures indicate significance.

$€=$ groups compared by Fisher's exact test for categorical variables.

* Statistical significance defined as $p \leq 0.05$.

${ }^{a}$ Median (interquartile range).

${ }^{\mathrm{b}}$ Arrival during the hours of 07:30-17:00.

${ }^{\mathrm{c}}$ Median time in minutes (interquartile range).

30 minutes of arrival (DTN $\leq 30)$ was also found to be significantly higher post-intervention $(p<0.001)$.

\section{Clinical Outcomes}

The analyses of clinical outcomes pre- versus post-intervention are illustrated in Table 2.
In-hospital mortality decreased significantly, from $18.6 \%$ pre-intervention to $9 \%$ post-intervention $(p=0.013)$. We also analyzed clinical outcomes of all study patients with DTN $\leq 60$ minutes versus DTN $>60$ minutes. Patients with DTN $\leq 60$ minutes had a lower rate of in-hospital mortality than those with a DTN $>60$ minutes $(p=0.044)$. The incidence of SICH within 36 hours and life-threatening bleeds were similar pre- and post-intervention. 
Table 2: Clinical outcomes of patients with acute ischemic stroke who received rt-PA

\begin{tabular}{|c|c|c|c|}
\hline Outcome & Pre-intervention $(\mathbf{n}=\mathbf{1 0 2})$ & Post-intervention $(\mathbf{n}=\mathbf{2 2 2})$ & p-values* \\
\hline LOS, days ${ }^{a}$ & $10(5-22)$ & $5(2-10)$ & 0.004 \\
\hline \multicolumn{4}{|l|}{ Mortality } \\
\hline In-hospital mortality $n(\%)$ & $19(18.6)$ & $20(9)$ & 0.013 \\
\hline 7 days in-hospital mortality $n(\%)$ & $10(9.8)$ & $14(6.3)$ & 0.264 \\
\hline \multicolumn{4}{|l|}{ Discharge location } \\
\hline Home $n(\%)$ & $30(29.4)$ & $74(33.3)$ & 0.483 \\
\hline $\begin{array}{l}\text { Favorable versus unfavorable } \\
\text { discharge location } n(\%)\end{array}$ & $77(75.5)$ & $195(87.8)$ & 0.005 \\
\hline \multicolumn{4}{|l|}{ Ambulatory status at discharge } \\
\hline Independent at discharge $n(\%)$ & $33(32.4)$ & $75(33.8)$ & 0.845 \\
\hline SICH $\leq 36$ hours $n(\%)$ & $5(5)$ & $19(8.6)$ & $0.360 €$ \\
\hline Life-threatening hemorrhage $n(\%)$ & 0 & $4(1.8)$ & $0.314 €$ \\
\hline
\end{tabular}

LOS = length of stay; SICH = secondary intracranial hemorrhage.

Groups compared by $\chi^{2}$ test for categorical variables and $t$-test for continuous variables.

Bold figures indicate significance.

$€=$ groups compared by Fisher's exact test for categorical variables.

*Statistical significance defined as $p \leq 0.05$.

${ }^{\mathrm{a}}$ Median (interquartile range).

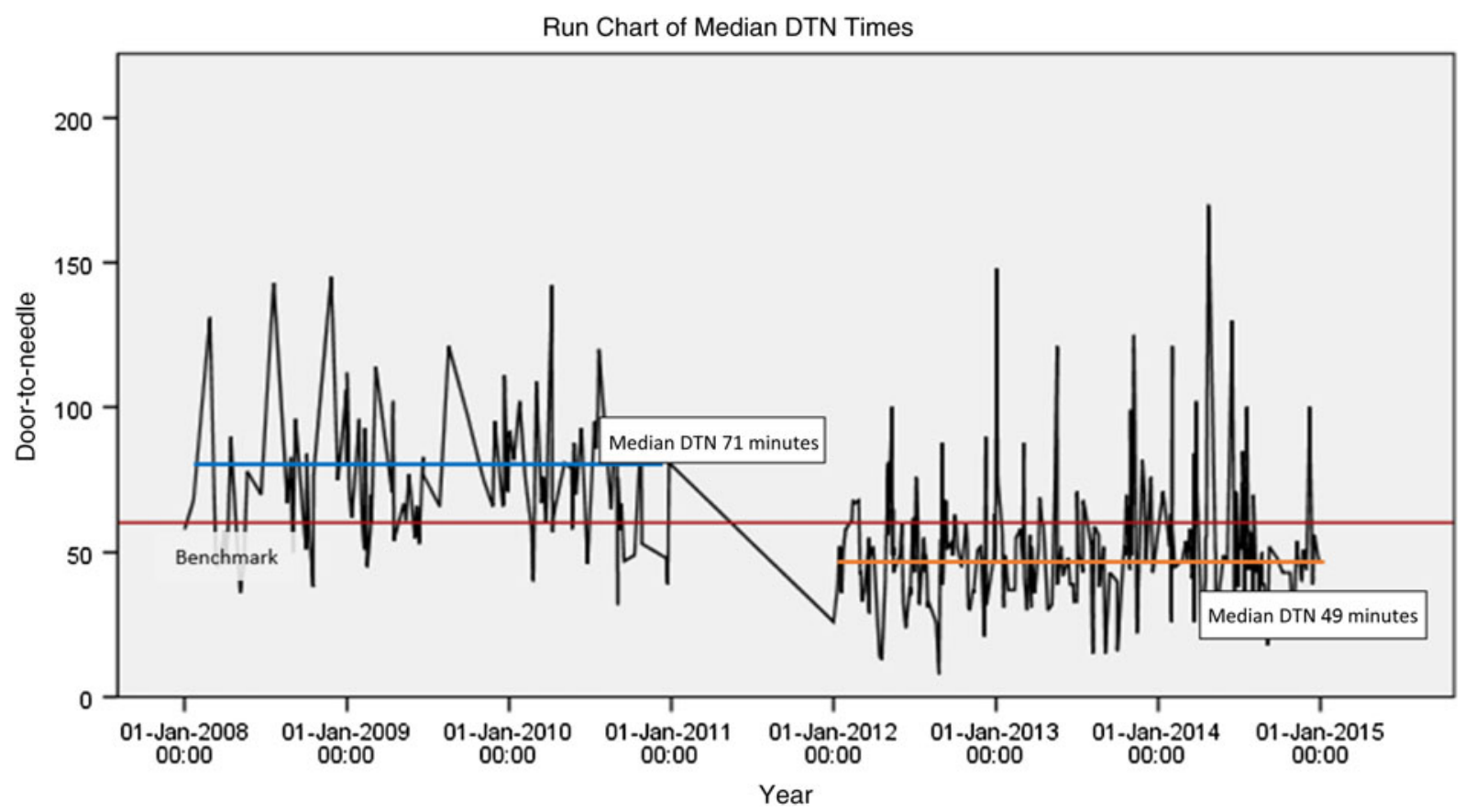

Figure 1: Run time chart of median DTN times of all study cases. Note: 2011 was considered the washout year, as the intervention took place in 2011; red horizontal line $=D T N$ national bench mark of $\leq 60$ minutes.

Discharge location was recorded as either home, acute rehabilitation, community hospital, long-term care facility (LTC), or death. Figure 2 provides a visual depiction of the percentage of patients in each of these categories pre- and postintervention. Of note, fewer patients were discharged to a LTC facility (3.2\% vs. $5.9 \%)$ and there were less in-hospital deaths (9\% vs. $18.6 \%$ ) post-intervention. Due to organizational changes during the study period which resulted in repatriation of patients to their catchment hospital more quickly after receiving rt-PA, significantly fewer patients went directly to acute rehabilitation facilities $(8.1 \%$ vs. $32.4 \%)$ and more patients went to community hospitals $(46.4 \%$ vs. $13.7 \%)$ post-intervention. Discharge location was also analyzed as the percentage of patients "discharged home" versus discharged to other locations. Our study found that the difference in the percentage of patients "discharged home" versus discharged to 

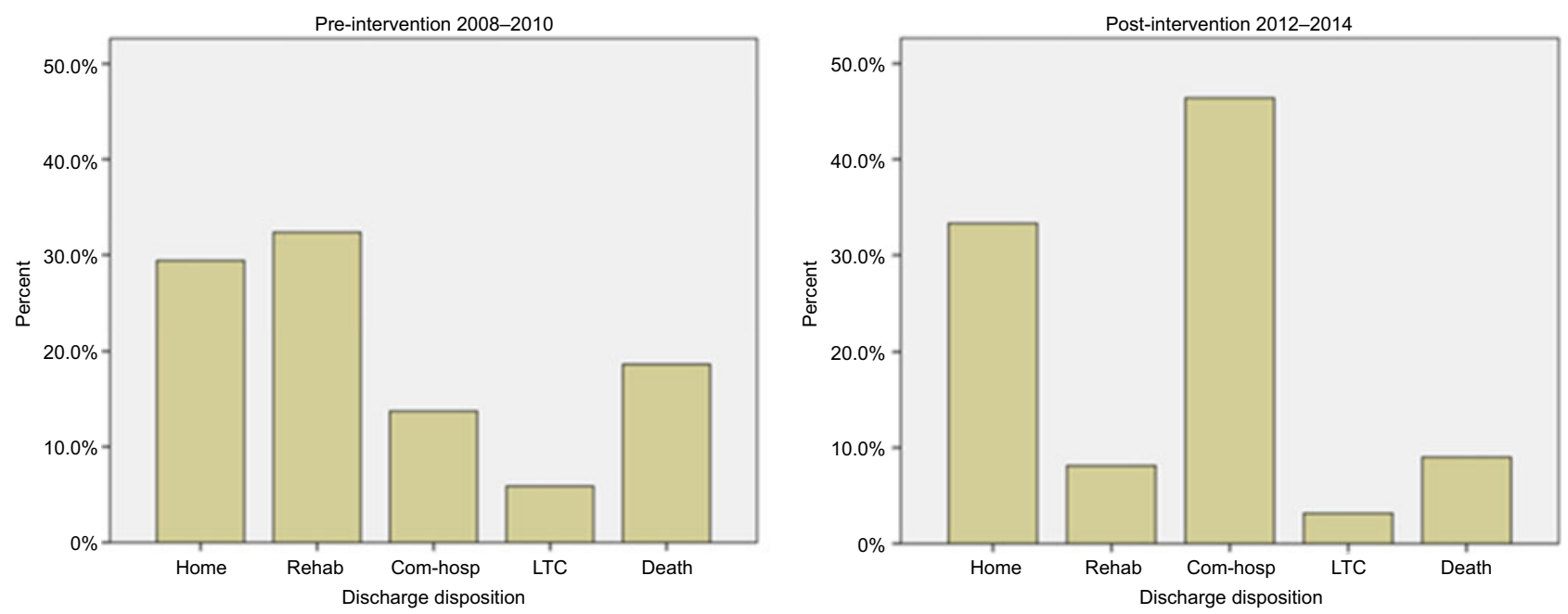

Figure 2: Discharge location: pre-versus post-intervention.
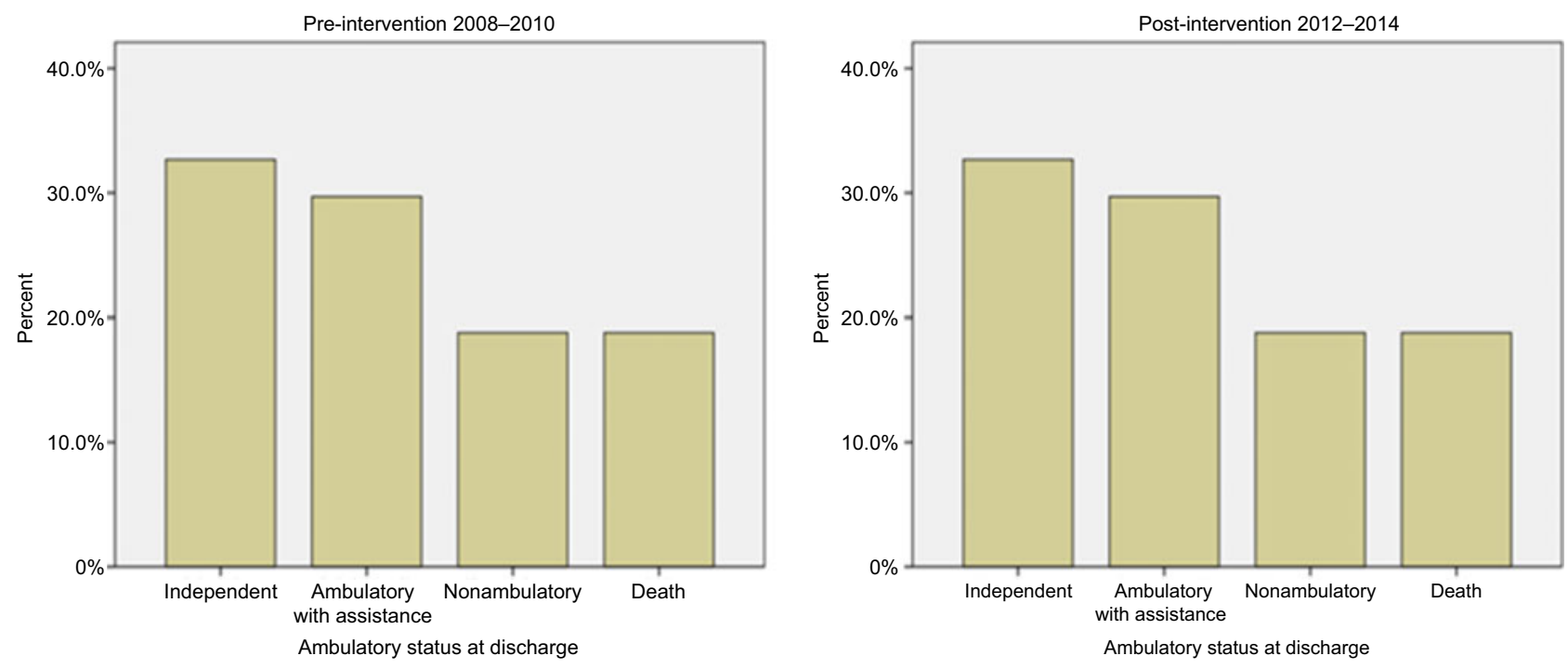

Figure 3: Ambulatory status upon discharge: pre- and post-intervention.

other locations pre- versus post-intervention was nonsignificant. Discharge was also analyzed in the categories of favorable (i.e., home, acute rehabilitation, and community hospital) versus unfavorable (i.e., LTC and death). This analysis revealed significantly more discharges to favorable locations in the postintervention group ( $p=0.005$; see Table 2). Finally, ambulatory status upon discharge was analyzed pre- and post-intervention. Categories included independent, which represented those who did not require any form of aid; with assistance, which included patients who required physical assistance; nonambulatory, which represented those who could not ambulate; and death. Figure 3 illustrates the percentage of patients in each category preand post-intervention. The results of a between-group analysis of independent versus all other categories were nonsignificant.

Finally, improvements at the organizational level were also evident in the post-intervention period. Median length of stay was reduced from 10 to 5 days $(p=0.004)$ in the post-intervention period. Moreover, more patients had access to treatment with rt-PA, as there were twice as many patients in the post-intervention group as compared to the pre-intervention group $(68.5 \%$ vs. $31.5 \%)$.

\section{Discussion}

Despite the limitations inherent in retrospective chart review research, this study contributes to the literature on timely hyperacute stroke care. The purpose of our study was to explore how the structural changes and the resulting dramatic process improvements impacted patient outcomes. The results provide convincing evidence that timely hyperacute stroke care contributed to a reduction in in-hospital mortality and an increase in the proportion of favorable discharges in the post-intervention period. Our quality improvement initiative also enabled twice as many patients to be treated with rt-PA following the quality improvement initiative. 


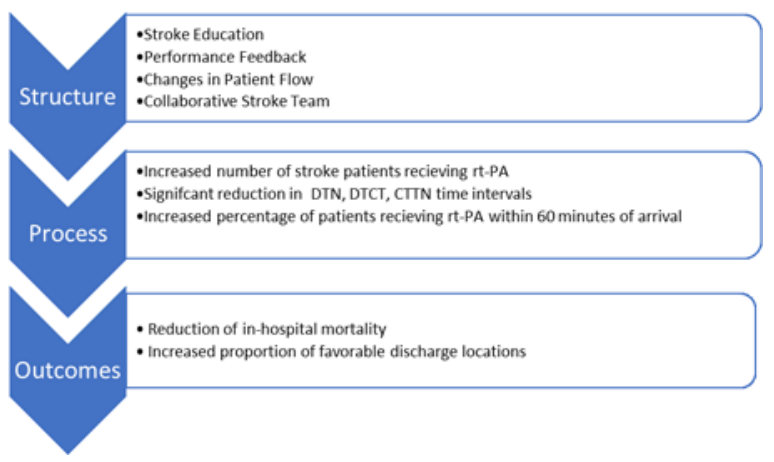

Figure 4: Summary of results: structural changes that led to improvements in the processes of care, which in turn led to improved clinical outcomes for acute stroke patients at the HSC Adult ED.

The Donabedian framework provided an appropriate and systematic approach to this research, including an organizational context for the discussion of the findings (see Figure 4).

\section{Structure and Process}

According to the Donabedian model, structure includes the patient population. Our sample size was relatively small, and data were elicited from a single center; therefore, the study findings may not be generalizable to other institutions or other settings. However, our findings concur with previous studies that have similar population characteristics. For example, Ganesh et al.'s (2014) ${ }^{4}$ large Canadian study $(\mathrm{N}$ weighted $=2049.5$ ) was comprised of $\sim 54.5 \%$ male and $67.1 \%$ with hypertension. Our population was also similar to Fonarow's $(2014)^{7}$ large study $(n=71,169)$, in which the median age was 72 years, with approximately $50 \%$ being male and $72.4 \%$ having hypertension. Unfortunately, we were not able to compare NIHSS scores pre- and post-intervention as our study revealed the NIHSS was only documented in $2 \%$ of cases pre-intervention and $28 \%$ of cases post-intervention. However, these abysmal statistics validated the need for our institution to develop more effective strategies for the consistent use of the NIHSS in clinical practice.

The structural quality improvement project changes responsible for improving the processes of care in our facility included more efficient patient flow, and acute stroke education with performance feedback for all ED staff. Using the principles of Lean ${ }^{10}$ to develop a VSM, inefficiencies were identified, nonvalue-added activity (waste) was removed from the stroke patient's pathway, and more effective/efficient workflow changes were adopted. Investing in a VSM was not only cost-effective but it also fostered the effectiveness of our multidisciplinary stroke team. Others ${ }^{13}$ have utilized the VSM strategy with similar dramatic improvements in process metrics.

A proficient, educated stroke team has been previously linked to providing improved processes in care and improved outcomes. ${ }^{14}$ Therefore, education was the second component of our process improvement. This stroke education likely improved the overall care provided to stroke patients while in the ED in the post-intervention phase, which, in turn may have also had a positive impact on patient outcomes. For example, earlier BP management was achieved due to increased awareness of its importance. Although education sessions were delivered to all ED staff as part of our initiative, implementing strategies for ongoing staff education are also important due to staff turnover and possible protocol drift, which could have a negative impact on adherence/compliance and the long-term success of the initiative.

Timely performance feedback is also central to stroke program success. ${ }^{7,14-16}$ While performance feedback provides an opportunity to celebrate success, addressing suboptimal performance with the entire stroke team was likely central to our improved program processes and patient outcomes. It follows that ongoing data collection and performance feedback will be necessary for our program to achieve the new national benchmark of DTN within 30 minutes. ${ }^{1}$ Our findings also suggest that the relatively easy data collection of process metrics may be a more effective use of scarce resources than collecting outcome indicator data for ongoing quality assurance program evaluation.

Our three-pronged approach focus on workflow, education, and performance feedback resulted in the formation of a collaborative, multidisciplinary stroke team with a common goal: to deliver best practice hyperacute stroke care. The team's enthusiasm was palpable when a stroke protocol patient arrived in our $\mathrm{ED}$, as everyone worked collaboratively to achieve a faster DTN time. Our approach resulted in a notable improvement in access to care, as evidenced by the significant increase in the number of patients in the post-intervention period. An example of the collaborative team in action was the significant reduction in median DTCT. This process improvement may be attributed to physical changes in the ED to reduce patient transport time, as well as staff working together to achieve a common goal. Similarly, we also observed a significant reduction in median CTTN. One could argue that our neurologists, who are responsible for determining rt-PA eligibility, had the biggest impact on reducing the CTTN time interval in the post-intervention period. Presentations to the neurologists on our suboptimal performance on key quality stroke indicators likely contributed to the patient assessments regarding rt-PA eligibility occurring much more quickly. Thus, ongoing education and feedback to the medical stroke team on reducing these time intervals is critically important.

The structures and processes of hyperacute stroke care at our center were clearly complex and intertwined. The reduction in DTN is never solely based on one improvement, but likely the combination of multiple factors, which lends support to multicomponent interventions, rather than addressing isolated issues. ${ }^{17}$ Therefore, a multidisciplinary systems approach is ideal. Several single-center studies, ${ }^{13,14,17,18}$ as well as multicenter studies, ${ }^{7,19}$ have demonstrated dramatic improvements in processes and patient outcome measures following a quality improvement initiative. However, quality improvement initiatives like ours are likely underrepresented in the literature, since outcome measures are more challenging to collect and less receptive to change than process metrics.

\section{Clinical Outcomes}

While our observational study revealed improvements in clinical and organizational outcomes that were most likely associated with structural and process changes, these positive changes may have also been influenced by other variables. However, the decrease in in-hospital mortality and increase in favorable discharge locations post-quality improvement project were likely 
due to the deliberate steps taken to improve overall acute stroke care at our institution.

While the differences in our 7-day mortality rates between the pre- and post-intervention groups $(6.3 \%$ and $9.8 \%$, respectively) were not statistically significant, these rates are comparable to the national Canadian average of $7.2 \%$ reported by Ganesh et al. $(2014)^{4}$ for patients receiving rt-PA at comprehensive stroke centers. It is important to acknowledge that factors such as stroke severity and neurological deterioration also impact 7-day mortality. Our higher 7-day stroke mortality rate post-intervention may be explained by the fact that patients were repatriated to community hospitals soon after they were deemed stable in the post-intervention phase, leaving the more critically ill patients who were at higher risk of mortality at our institution.

Our in-hospital mortality was significantly reduced postquality improvement initiative. The sharp drop in median DTN time was likely responsible for this significant reduction; however, it may also be due to the overall improvement in stroke care delivered in our ED post-intervention, such as earlier assessment, diagnostics, and BP management. We also analyzed in-hospital mortality rates of all study patients who received rt-PA within 60 minutes of arrival $(\mathrm{DTN} \leq 60)$ and found a significantly lower in-hospital mortality compared to patients with DTN $>60$ minutes $(p=0.044)$. These findings concur with others $^{20}$ who have reported similar results, and thus support the contention that timely care does reduce mortality.

Possible adverse events linked to thrombolytic therapy include life-threatening bleed and SICH. The similarity in the rates of adverse events between pre- and post-intervention suggests that patient safety was not jeopardized by faster care. A major reason for these favorable findings is likely due to the thorough screening by the neurologists when assessing a potential thrombolytic candidate. However, it is important to note our post-intervention SICH of $8.6 \%$ was greater than the target of $<6 \%$ in the Canadian Stroke Best Practice Recommendations. ${ }^{21}$ Our higher post-intervention SICH rate is likely due to the larger number of patients in the post-intervention group (222 vs. 102 patients) and the longer median OTD time in the postintervention phase ( 84 vs. 64 minutes). Treating patients late in the thrombolytic window is associated with an increased risk of SICH. ${ }^{8,20}$ It is critically important to complete a timely and comprehensive assessment of rt-PA inclusion/exclusion criteria, while making every effort to treat eligible patients early in the thrombolytic window.

Stroke is the number one cause of adult disability ${ }^{1}$; therefore, measuring functional outcomes is critically important to gaining insight into the stroke trajectory and developing strategies for optimal rehabilitation. Numerous studies have reported discharge location $^{7,8,13,18-20}$ and ambulation upon discharge, ${ }^{7,8,20}$ as proxies of functional outcomes, as these data are relatively easy to collect. Although several larger studies ${ }^{7,8}$ have demonstrated a significant increase in the number of stroke patients being independent at discharge post-DTN quality improvement project, our findings were inconclusive. These disparate findings may be explained by the relatively small number of patients in our investigation. However, in the post-intervention phase, we discovered that patients were repatriated much sooner to their community hospital if medically stable. The patients who then remained at our facility likely either had an excellent outcome and were discharged home very early post-rt-PA therapy or were more complex cases requiring tertiary care at our facility.

In our study, discharge location was captured in the following categories: home, acute rehabilitation, community hospital, LTC, and death. Each of these categories is associated with a certain level of independence or dependence, with discharge home being an important indicator of independence and full recovery. Our results demonstrated that a lower proportion of patients died or went to LTC post-intervention. We also compared unfavorable discharge locations (i.e., death or LTC placement) with favorable discharge locations (i.e., home, acute rehab, and community hospital). Akin to others, ${ }^{13}$ significantly more patients were discharged to a favorable discharge location post-quality improvement initiative. These findings are encouraging for two reasons: (1) fewer transfers to LTC result in healthcare cost reduction; and (2) since the brain recovers for a long-time post-stroke, it is vital that patients access rehabilitation at either acute rehabilitation facilities or community hospitals to maximize their recovery. The overall improvement of stroke care at our hospital was likely an influencing factor in the increased number of favorable discharges. Every individual case where a good functional outcome prevails is a personal victory for patients and their families.

\section{LIMITATIONS}

Despite the inherent limitations of a retrospective chart review investigation, our study was cost-effective and generated a large amount of important observational data for our center. Generalizability of the findings of this investigation may be limited due to the small sample size and the fact that it was a single-center study. However, our sample characteristics were similar to a large population-based study in which Ganesh et al. ${ }^{4}$ explored ischemic stroke in Canada. It is important for future studies to aim for larger sample sizes and to include all patients who were repatriated. While the three distinct interventions (i.e., stroke education, performance feedback, and workflow improvements) may be a confounding factor, as we could not isolate the impact of each of these changes, the three components of the intervention could be relatively easily replicated in future studies.

There were also several limiting factors related to instrumentation. The current study did not measure stroke impairment with a reliable and validated stroke deficit tool, such as the NIHSS, which has been consistently used in other similar studies. $7,8,13,17,18,20,22$ However, discharge home and ambulation at discharge which have been used in previous studies ${ }^{7,8,13,20}$ as measures of functional outcomes were included. In the post-intervention phase, stroke patients were repatriated soon after thrombolysis, which may have skewed our data. Unfortunately, we did not have access to any outcome measures for the patients who were repatriated to community hospitals in the early phase post-rt-PA. A future study linking administrative data will be considered. Finally, to our knowledge, there were no concurrent stroke initiatives taking place on the inpatient units; however, other unidentified confounding variables may also have influenced the clinical outcomes of patients in the current study. Lastly, our study only included those patients that received rt-PA. Exploring if our quality improvement initiative also improved outcomes for stroke patients not eligible for rt-PA during the study period would have proved interesting. 


\section{Conclusions}

As a leading cause of death and disability, stroke continues to significantly impact too many Canadians, underscoring the need to continuously strive to deliver faster, safer, and better care. Our study has shown that DTN quality improvement initiatives can successfully reduce median DTN time and enable a greater number of patients to benefit from thrombolytic therapy. More importantly, we have shown that the dramatic process improvements likely translated into a reduction in in-hospital mortality and an increase in favorable discharges. This study has provided the foundation for future stroke process improvement research at our institution and also highlights the need for all organizations to invest in strategies that ensure optimal time-driven hyperacute stroke care.

\section{ACKNowledgments}

The corresponding author would like to acknowledge the Health Sciences Centre - Dolly \& Michael Gembey Nursing Award for providing support for this thesis research study.

\section{Disclosures}

$\mathrm{SA}$ and KD are employees of the HSC. The other authors do not have anything to disclose.

\section{STATEMENT OF AUTHORSHIP}

SA contributed to the study design, data collection, data analysis, data interpretation, and drafting and editing of this manuscript. JVS provided guidance during the entire process of this thesis study. TS contributed to data analysis and data interpretation. KD contributed to the study concept and data interpretation. All authors contributed to the development and editing of this manuscript and approved the final version.

\section{REFERENCES}

1. Boulanger JM, Lindsay MP, Gubitz G, et al. Canadian stroke best practice recommendations for acute stroke management: prehospital, emergency department, and acute inpatient stroke care, update 2018. Int J Stroke. 2018;13(9):949-84.

2. Statistics Canada. Population estimates on July 1st, by age and sex; 2018. Retrieved from https://www150.statcan.gc.ca/t1/tbl1/en/ tv.action?pid=1710000501; accessed August 2019.

3. Saver JL. Time is brain - quantified. Stroke. 2006;37:263-6.

4. Ganesh A, Camden M, Lindsay P, et al. The quality of treatment of hyperacute ischemic stroke in Canada: a retrospective chart audit. CMAJ. 2014;2:E233-9.

5. Heart and Stroke Foundation. Access to stroke care: the critical first hours; 2015. Retrieved from https://www.heartandstroke. ca/-/media/pdf-files/canada/stroke-report/hsf-stroke-report-2015. ash $x$ ?la=en\&hash $=5484731938$ A116C95008934CF76D445ED9 F60546; accessed January 2020.

6. Powers WJ, Rabinstein AA, Ackerson T, et al. 2018 guidelines for the early management of patients with acute ischemic stroke. A guideline for healthcare professionals from the American Heart Association/American Stroke Association. Stroke. 2018;49: e46-99.

7. Fonarow GC, Zhao X, Smith E, et al. Door-to-needle for tissue plasminogen activator administration and clinical outcomes in acute ischemic stroke before and after a quality improvement initiative. JAMA. 2014;311(16):1632-40.

8. Saver JL, Fonarow GC, Smith EE, et al. Time to treatment with intravenous tissue plasminogen activator and outcome from acute ischemic stroke. JAMA. 2013;309(23):2480-8.

9. Wardlaw JM, Murray V, Berge E, et al. Recombinant tissue plasminogen activator for acute ischemic stroke: an updated systematic review and meta-analysis. Lancet. 2012;379:2364-72.

10. Donabedian A. The quality of care. JAMA. 1988;260(12):1743-8.

11. Ghrooda E, Alcock S, Jackson AC. Improvement in thrombolytic therapy administration in acute stroke with feedback. Can J Neurol Sci. 2012;39:789-92.

12. Lean Enterprise Institute. Principles of lean; 2018. Retrieved from https://www.lean.org/WhatsLean/Principles.cfm; accessed September 2018.

13. Ford A, Williams JA, Spencer M, et al. Reducing door-to-needle times using Toyota's lean manufacturing principles and value stream analysis. Stroke. 2012;43:3395-8.

14. Ruff IM, Ali SF, Goldstein JN, et al. Improving door-to-needle times: a single centre validation of the target stroke hypothesis. Stroke. 2014;45:504-8.

15. Casaubon LK, Boulanger JM, Blacquiere D, et al. Canadian stroke best practice recommendation: hyperacute stroke care guidelines, update 2015. Int J Stroke. 2015;10(6):924-40.

16. Saver JL, Smith EE, Fonarow GC, et al. (2010). The "Golden Hour" and acute brain ischemia. Stroke. 2010;41:1431-9.

17. Meretoja A, Strbian D, Mustanoja S, Tatlisumak T, Lindsberg P, Kaste M. Reducing in-hospital delay to 20 minutes in stroke thrombolysis. Neurology. 2012;79:306-13.

18. Busby L, Owada K, Dhungana S, et al. Code fast: a quality improvement initiative to reduce door-to-needle times. J Neurointervent Surg. 2016;8(7):661-4.

19. Song S, Fonarow GC, Olson DM, et al. Association of get with the guidelines-stroke program participation and clinical outcomes for medicare beneficiaries with ischemic stroke. Stroke. 2016;47:1294-302.

20. Fonarow GC, Smith EE, Saver JL. et al. Timeliness of tissue-type plasminogen activator therapy in acute ischemic stroke: patient characteristics, hospital factors, and outcomes associated with door-to-needle times within 60 minutes. Circulation. 2011;123:750-8.

21. Heart and Stroke Foundation. Quality of stroke care in Canada. Stroke key quality indicators and stroke case definitions; 2016. Retrieved from http://ontariostrokenetwork.ca/wp-content/ uploads/2016/09/2016-StrokeCaseDefn-KQI-Update-EN-FINALSept2016.pdf?a2ca24; accessed September 2018.

22. Van Schaik SM, Scott S, de Lau LML, et al. Short door-to-needle times in acute ischemic stroke and prospective identification of its delaying factors. Cerebrovasc Dis. 2015;5:75-83. 